\title{
Significance of AKT in gastric cancer (Review)
}

\author{
TAKAMITSU SASAKI $^{1}$ and HIROKI KUNIYASU ${ }^{2}$ \\ ${ }^{1}$ Department of Gastroenterological Surgery, Fukuoka University School of Medicine, Jonan-ku, Fukuoka 814-0180; \\ ${ }^{2}$ Department of Molecular Pathology, Nara Medical University, Kashihara, Nara 634-8521, Japan
}

Received July 17, 2014; Accepted September 2, 2014

DOI: $10.3892 /$ ijo.2014.2678

\begin{abstract}
AKT is a serine/threonine kinase activated downstream of integrin, which is a receptor for various pro-proliferation and bioactive substances as well as the extracellular matrix receptor. The activation of AKT often contributes to tumorigenesis and plays a role in regulating cell motility, which is critical for local invasion and metastasis. In recent years, it has also drawn attention as a target of new molecular-targeted agents. When analyzing the role of AKT in cancer cells, it is important to consider the microenvironment and variations of cancers. In this review, a basic overview of the role of AKT in normal and malignant tissues is provided, along with an in-depth discussion of its role in gastric cancer and its potential as a target for therapy.
\end{abstract}

\section{Contents}

1. Introduction

2. What is AKT?

3. AKT and the pro-survival effect

4. AKT signal transduction in cancer cells

5. The activion of various cancer cells by AKT

6. The PI3K-AKT-mTOR pathway and its activation in gastric cancer

7. hTERT

8. The concurrent effects of pAKT and hTERT on the progression of gastric cancer

9. Relationships among the levels of pAKT and iNOS, NT and hTERT in gastric mucosa

10. Conclusion

Correspondence to: Dr Takamitsu Sasaki, Department of Gastroenterological Surgery, Fukuoka University School of Medicine, Nanakuma 7-45-1, Jonan-ku, Fukuoka 814-0180, Japan

E-mail: takamitu@fc4.so-net.ne.jp

Abbreviations: PI3K, phosphatidylinositol-3 kinase; hTERT, human telomerase reverse transcriptase; pAKT, phosphorylated AKT; EGF, epidermal growth factor; PTEN, phosphatase and tensin homolog deleted on chromosome 10; mTOR, mammalian target of rapamycin; NF, nuclear factor; EMT, epithelial-mesenchymal transition; VEGF, vascular endothelial growth factor

Key words: AKT, gastric cancer, human telomerase reverse transcriptase

\section{Introduction}

AKT signaling pathways are related to the suppression of apoptosis, proliferation and metastasis of cells, angiogenesis and various other processes (1). AKT is involved in the proliferation of cells via its phosphorylation of Cyclin D1, and promotes the phosphorylation of BAD and caspase-9 which is associated with apoptosis. By inhibiting these molecules (BAD and caspase-9), AKT suppresses apoptosis. AKT plays an important role in the chemosensitivity to various agents, because it prevents the normal apoptotic response to treatment. AKT is also deeply involved in angiogenesis and the invasion of cancer cells into surrounding tissues through vascular endothelial growth factor (VEGF) and MMP $(2,3)$. The mechanism regulating phosphatidylinositol-3 kinase (PI3K)/AKT has been shown to be abnormal in various solid carcinomas and hematological neoplasms. The overexpression of AKT at the DNA or protein level has been reported in breast cancer and stomach cancers (1); thus, AKT has drawn attention as a target for the development of new molecular-targeted cancer therapeutic agents. In this review, a basic overview of the role of AKT in normal and malignant tissues is provided, along with an in-depth discussion of its role in gastric cancer and its potential as a target for therapy.

\section{What is AKT?}

AKT is a serine/threonine kinase activated at downstream of integrin, which is a receptor for various pro-proliferation and bioactive substances (receptor tyrosine kinases and $\mathrm{G}$ protein-coupled receptors) as well as extracellular matrix receptor. AKT has three isoforms, with AKT1 and AKT2 being expressed ubiquitously, while the expression of AKT3 occurs in limited tissues (brain and testes). All three isoforms are composed of a pleckstrin homology $(\mathrm{PH})$ domain, linker domain, kinase domain and C-terminal hydrophobic domain. Following the activation of PI3 kinase, the phosphorylation of both threonine 308 and serine 473 through PDKI and mTORC2 is required for complete activation of AKT $(4,5)$. Activated AKT phosphorylates various substrates to exert its functions in cell proliferation, growth, anti-apoptosis and cell cycle progression.

\section{AKT and the pro-survival effect}

In cancer cells, the activation of various receptors occurs as a result of stimulation from proliferators and adhesion to 
the extracellular matrix, and AKT is subsequently activated downstream of PI3 kinase. As noted above, the PI3K/AKT signaling pathway is involved in the suppression of apoptosis, the proliferation and metastasis of cells, as well as in angiogenesis (1). AKT regulates cell proliferation by phosphorylating Cyclin D1 and promotes the phosphorylation of BAD and caspase-9, which are associated with apoptosis. By inhibiting the functions of these proteins, AKT suppresses apoptosis, clearly showing that AKT plays an important role in chemosensitivity, because many chemotherapeutic agents exert their effects by inducing caspase-mediated apoptosis. AKT is also deeply involved in angiogenesis and the invasion of cancer cells into surrounding tissues through VEGF and MMP (2,3). In the phosphatase and tensin homolog deleted on chromosome 10 (PTEN) heterozygous knockout mice, $\mathrm{PI} 3 \mathrm{~K} / \mathrm{AKT}$ signaling is activated continually, which causes tumor formation (6).

\section{AKT signal transduction in cancer cells}

AKT is a protein in the PI3K pathway. Stimulation of receptor tyrosine kinases or G-coupled proteins activates PI3K, which in turn activates AKT. AKT phosphorylation is maintained by heat shock protein 90, and AKT is dephosphorylated by protein phosphatase $2 \mathrm{~A}$. Thus, AKT is involved in signaling mediated by various growth factors and cytokines. In particular, insulin-like growth factor 1 (IGF-1), epidermal growth factor (EGF) receptor and human EGF receptor 2, which are important in cancer progression, activate $\operatorname{AKT}(7,8)$. Based on these functions, AKT activation or overexpression can serve as a biomarker for predicting the metastasis of human gastrointestinal cancer (9).

The phosphorylation of AKT modulates signals from PTEN, and the mammalian target of rapamycin (mTOR) to exert diverse effects on cells (10). In this regard, AKT1 is recognized as an apoptotic inhibitor, which enhances cancer promotion. Phosphorylation via AKT inactivates Bcl-2 antagonist of cell death (Bad), resulting in its dissociation from Bcl-2. Nuclear factor $(\mathrm{NF}) \kappa \mathrm{B}$ is also activated by AKT, which in turn upregulates the transcription of many survival genes (11). AKT also induces angiogenesis through the upregulation of VEGF (12). The existence of an extensive AKT-microRNA regulatory network suggests that microRNA-mediated gene regulation interacts with the AKT signaling pathway (13). Hence, the expression of AKT is a pivotal tumorigenic factor, and $\mathrm{AKT}$ is recognized as a relevant molecular target for cancer therapy (8).

\section{The activion of various cancer cells by AKT}

It has been reported that AKT has some involvement in the motor activity of various cells, including cancer cells. For example, AKT increases the motor activity of mammary epithelial cells by increasing the expression of MMP-2 (14). In addition, an examination using cells derived from fibrosarcoma and pancreatic cancer showed that increased expression of the receptor for MMP-9 and IGF-1 led to enhanced metastasis of cells, and this effect depended on the activation of AKT kinase $(15,16)$. It was also reported that high expression of AKT induced epithelial-mesenchymal transition (EMT) and enhanced the invasive potential of squamous cell carcinoma cells (17). AKT gene amplification and mutations cause AKT activation in many solid carcinomas and hematological neoplasms, and mutations of PTEN, which has a negative effect on PI3 kinase and growth factor receptors upstream of AKT activation, occur frequently, which cause the abnormal activation of AKT $(5,18)$. AKT signaling consolidates various intercellular signals; hence, it is essential to understand the molecular mechanism for future studies of carcinogenesis and cancer therapy.

\section{The PI3K-AKT-mTOR pathway and its activation in gastric cancer}

mTOR is a serine/threonine kinase of $289 \mathrm{kD}$ that is activated by AKT and Rheb. mTOR exists as two separate complexes in the cytoplasm (TORC1 and TORC2). TORC1 contains an mTOR binding protein, Raptor, which facilitates the transcription and translation of the protein through phosphorylation of S6K1 (p70 S6 kinase 1 = 40S ribosomal protein S6 kinase 1) and eukaryotic translation initiation factor 4E-binding protein 1 (4EBP1) in the presence of amino acids, and accelerates cell proliferation. In addition, the activation of hypoxia-inducible factor-1 (HIF-1) leads TORC1 to induce angiogenesis through VEGF and in the facilitation of progression of the cell cycle from the G1 to S phase through Cyclin D. At the same time, TORC2, which contains Rictor, provides positive feedback to activate AKT, and facilitates cell survival.

The PI3K-AKT-mTOR pathway has been reported to be activated in many malignant tumors due to abnormalities in various genes such as EGFR, HER2, PTEN, PIK3CA and TSC1 (19,20). In gastric cancer, it was reported that gene mutations and gene amplifications of PIK3CA, AKT gene amplification and a loss of PTEN can all cause activation of the PI3K-AKT-mTOR pathway, and that such mutations or amplifications are present in 30-60\% of cases (21). Moreover, HIF-1 is involved in cell proliferation, angiogenesis and blood vessel maturation in gastric cancer. These fundamental findings suggest that the PI3K-AKT-mTOR pathway is important as a target for antineoplastic agents (22).

\section{7. hTERT}

The activity of human telomerase reverse transcriptase (hTERT) is regulated by its expression and phosphorylation. Protein kinase $\mathrm{C}$ and AKT can both phosphorylate hTERT $(23,24)$. AKT-mediated phosphorylation of hTERT induces the intranuclear translocation of hTERT, and subsequently, activates hTERT. In contrast, ring finger protein 1, an E3 ubiquitin ligase, decreases the activity of hTERT by inducing its ubiquitylation (25).

Dysregulated PTEN/PI3K/AKT signaling interacts with the Wingless-INT pathway to induce EMT, which is usually associated with a cancer stem cell-phenotype and a poor prognosis (26). It has recently been reported that hTERT promotes transforming growth factor- $\beta$ and $\beta$-catenin-induced EMT by inducing the nuclear translocation of $\beta$-catenin and increasing its transcriptional activity for vimentin expression (27). Therefore, PTEN/PI3K/AKT signaling enhances EMT and stem cell phenotypes. In one of our studies, significant correlations 

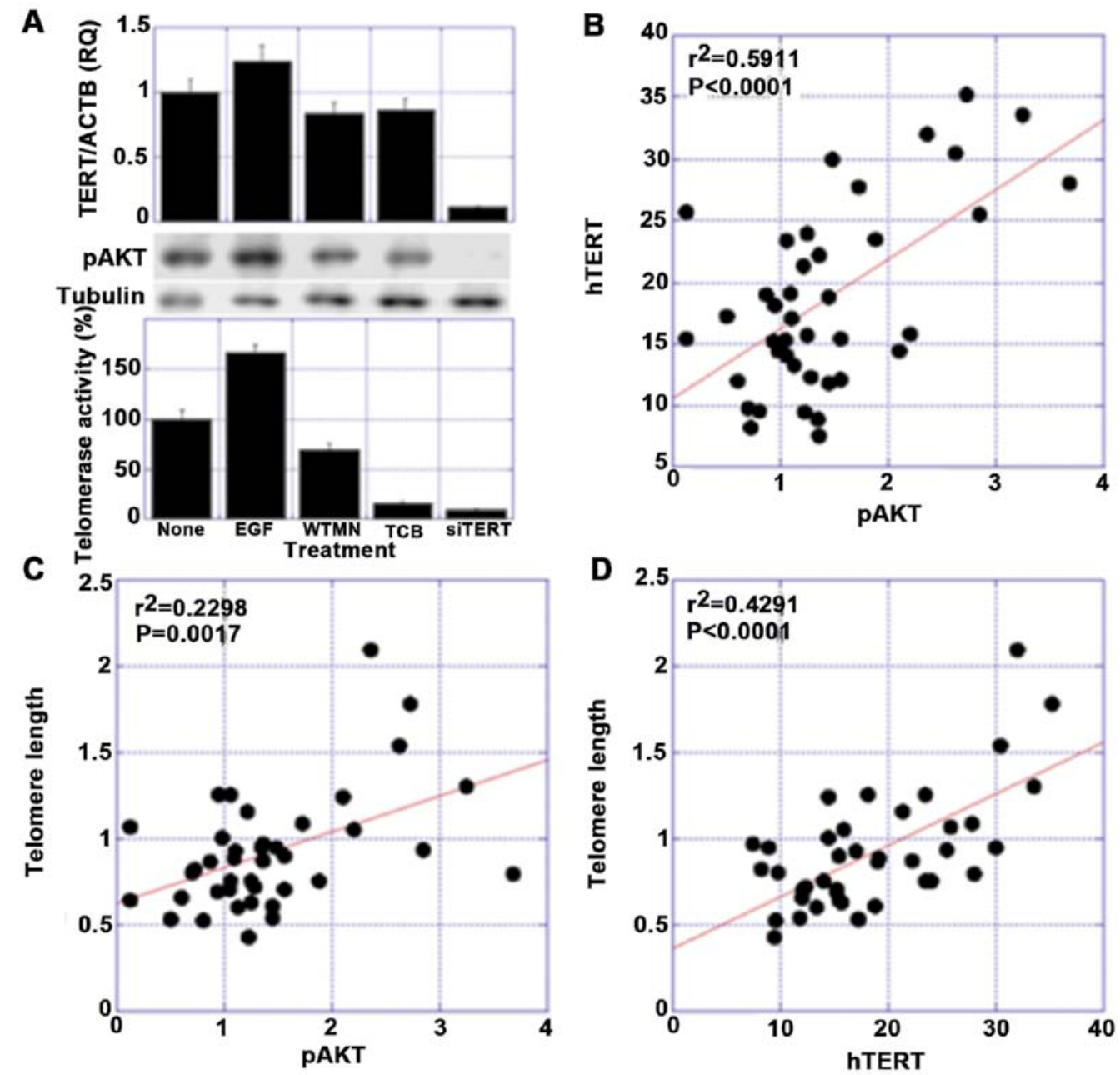

Figure 1. The relationships among phosphorylated AKT (pAKT), human telomerase reverse transcriptase (hTERT) and the telomerase activity. (A) The levels of the pAKT protein, hTERT mRNA and telomerase activity were compared in MKN28 cells treated with epidermal growth factor (EGF) (10 ng/ml), wortmannin $(10 \mathrm{nM})$, triciribine $(10 \mu \mathrm{M})$ and siRNA against hTERT. Tubulin was used as a loading control. (B-D) The levels of the pAKT protein, TERT mRNA and telomerase activity were compared in 40 gastric cancer tissue specimens.

were found among the levels of phosphorylated AKT (pAKT), hTERT expression and the telomere length (Fig. 1).

\section{The concurrent effects of pAKT and hTERT on the progression of gastric cancer}

In our study, the association of AKT phosphorylation, TERT expression and telomerase activity was confirmed in tissue specimens from 40 patients with gastric cancer. The survival rates of the pAKT-high patients or the pAKT-high and hTERThigh patients were significantly poorer than those in other patients. These associations resulted in poor prognoses in cases with high pAKT levels or high pAKT/hTERT levels. A multivariate analysis revealed that the pAKT levels or pAKT/hTERT levels were independent prognostic factors. The examination of more gastric cancer cases is required to confirm the hypothesis that the EMT/stem cell phenotype affects disease progression.

Angiogenesis is associated with cancer progression (28). VEGF expression is closely related to neovascularization and cancer progression in many malignancies. The PI3K/AKT pathway is one of the inducers of a VEGF response, which includes other inducers, such as mitogen-activated protein kinase (extracellular signal-regulated kinases or p38), Src, focal adhesion kinase, Rho family GTPases and endothelial nitric oxide (29). The PI3K/AKT pathway increases the secretion of VEGF from cancer cells by HIF-1-dependent and -independent mechanisms (30). Therefore, AKT suppression could result in an anti-angiogenic effect on gastric cancer.

Our data showed that AKT and hTERT were widely expressed in gastric cancer. The concurrent expression of these two proteins at high levels is associated with a poor prognosis (31). These results suggest that AKT and hTERT are good molecular targets, and that inhibiting them could be useful for the treatment of gastric cancer (Fig. 2).

\section{Relationships among the levels of pAKT and iNOS, NT and hTERT in gastric mucosa}

AKT phosphorylation was associated with the expression levels of inducible nitric oxide synthase (iNOS) and hTERT, and with increased levels of nitrotyrosine (NT). To establish the role of oxidative stress and v-akt murine thymoma viral oncogene homolog (AKT) activation in the development of gastric cancer, we examined the levels of pAKT, iNOS, NT and hTERT by an enzyme-linked immunosorbent assay in 73 non-cancerous gastric mucosa samples and 10 gastric carcinoma specimens (32). 

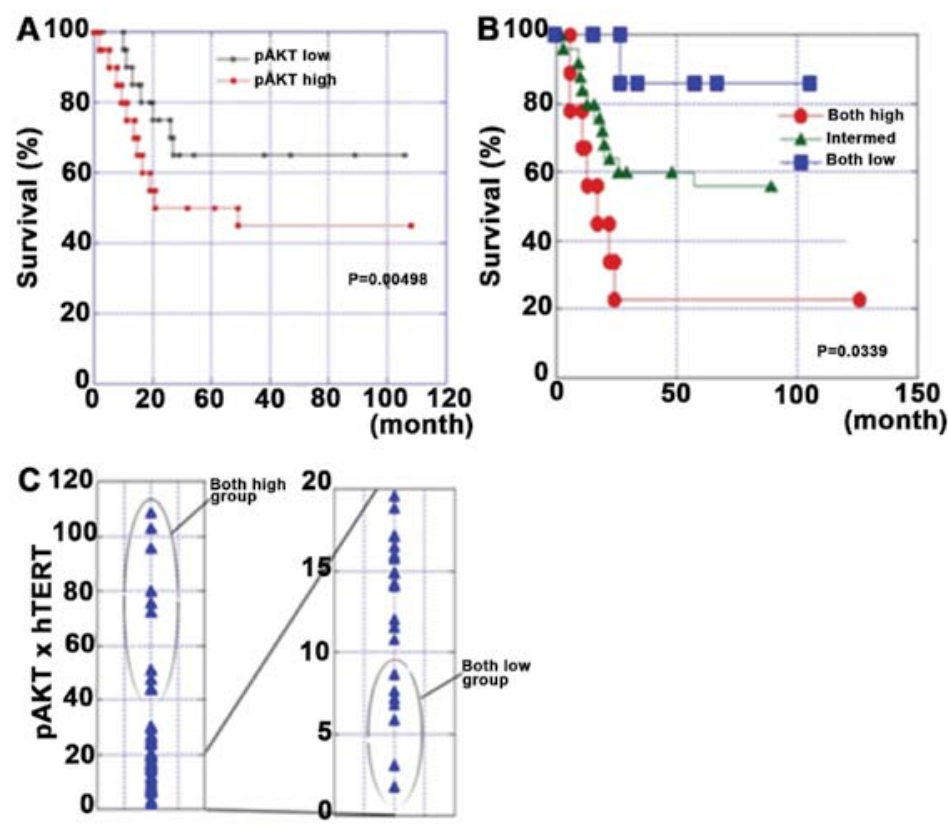

Figure 2. The results of the survival analyses of 40 gastric cancer cases. (A) The overall survival of the 20 cases with higher phosphorylated AKT (pAKT) levels (pAKT high) was compared with that of 20 cases with lower pAKT levels (pAKT low) using the Kaplan-Meier method. (B) The overall survival rates were compared among nine cases with high AKT and high TERT (Both high), seven cases with low pAKT and low TERT (Both low) and 24 cases with intermediate expression (Intermed) by using the Kaplan-Meier method. (C) The distribution of the products of the pAKT value by the human telomerase reverse transcriptase (hTERT) value in the both high group (left panel) and the both low group (right panel).

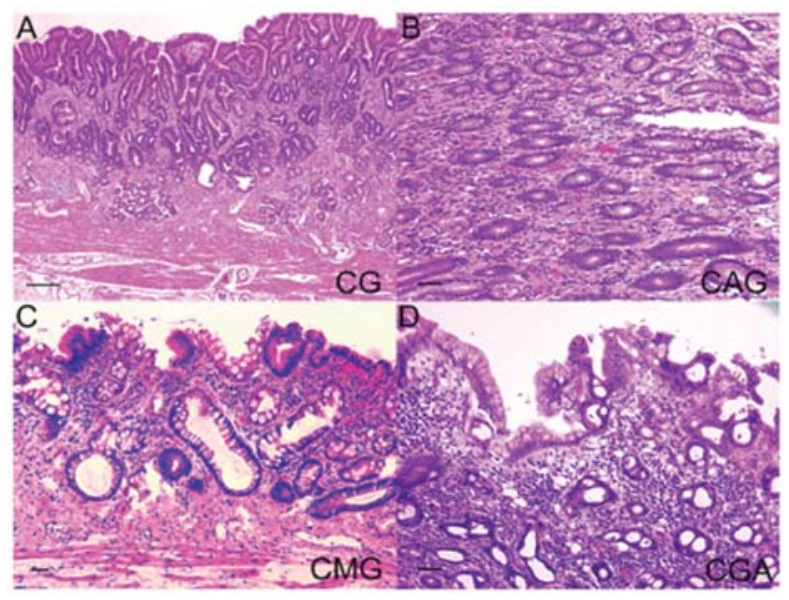

Figure 3. The histopathological findings of the gastric mucosa (hematoxylin and eosin staining). (A) Chronic gastric mucosa without Helicobacter pylori (H. pylori) (CG). (B) Chronic active gastritis with H. pylori (CAG). (C) Chronic metaplastic gastritis without H. pylori (CMG). (D) Chronic gastritis with atypia without $H$. pylori (CGA). Bar, $100 \mu \mathrm{m}$.
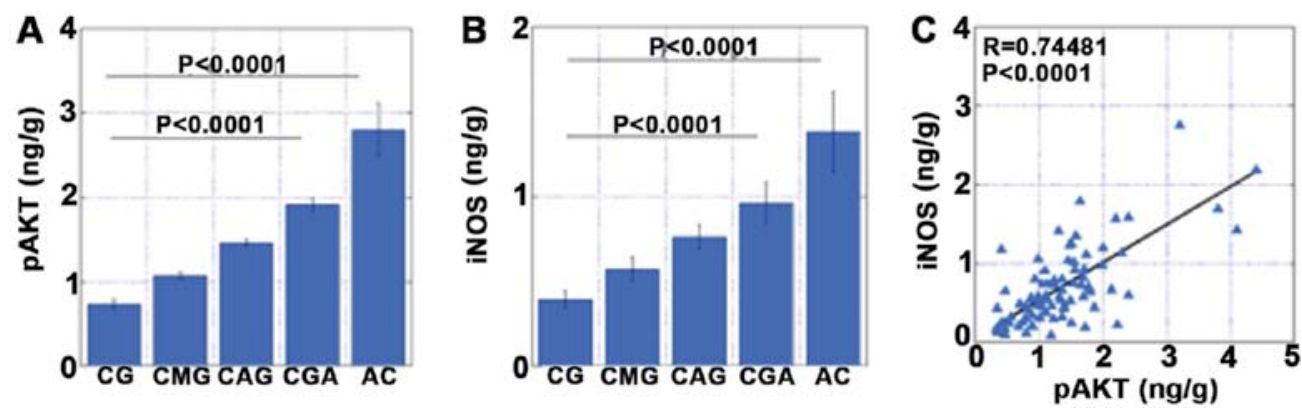

Figure 4. The level of phosphorylated v-akt murine thymoma viral oncogene homolog (AKT) and its association with inducible nitric oxide synthase (iNOS) expression. The levels of (A) phosphorylated AKT (pAKT) and (B) iNOS were examined by enzyme-linked immunosorbant assays. (C) The relationship between pAKT and iNOS was compared by performing a Spearman's correlation analysis. Error bar, SE. CG, chronic gastric mucosa without Helicobacter pylori (H. pylori); CAG, chronic active gastritis with $H$. pylori; CMG, chronic metaplastic gastritis without $H$. pylori; CGA, chronic gastritis with atypia without $H$. pylori. The levels of pAKT and iNOS (A and B) increased from CG to CMG to CAG to CGA and (C) were significantly correlated. 

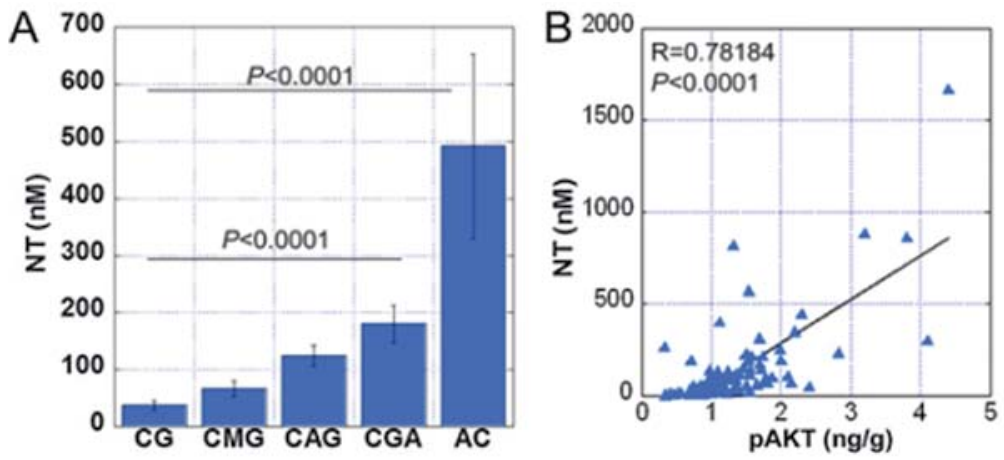

Figure 5. The nitrotyrosine (NT) levels and the association with phosphorylated v-akt murine thymoma viral oncogene homolog (AKT) expression. (A) The level of NT was examined by an enzyme-linked immunosorbant assay. (B) The relationship between NT and phosphorylated AKT (pAKT) was compared using a Spearman's correlation analysis. Error bar, SE. CG, chronic gastric mucosa without Helicobacter pylori (H. pylori); CAG, chronic active gastritis with H. pylori; CMG, chronic metaplastic gastritis without H. pylori; CGA, chronic gastritis with atypia without $H$. pylori. The levels of NT (A) increased in the order of $\mathrm{CG}<\mathrm{CMG}<\mathrm{CAG}<\mathrm{CGA}$ and $(\mathrm{B})$ were correlated with the levels of pAKT.
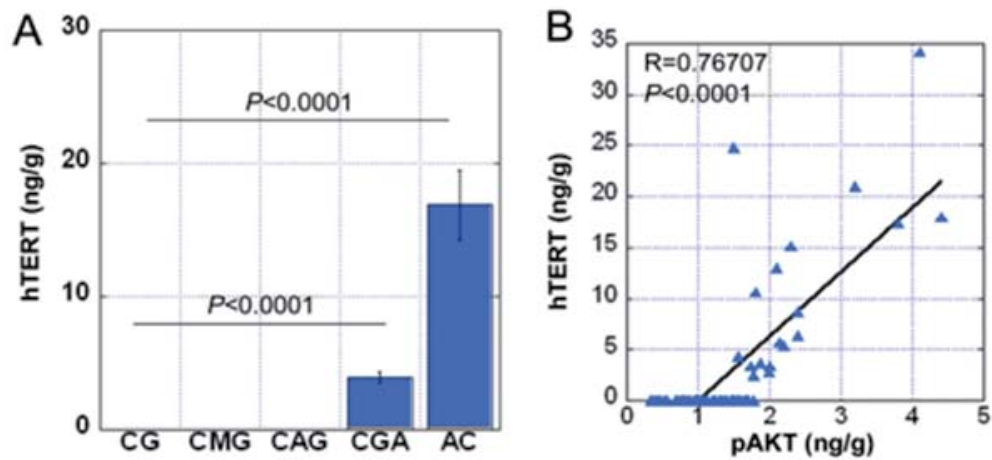

Figure 6. The human telomerase reverse transcriptase (hTERT) level and the association with phosphorylated v-akt murine thymoma viral oncogene homolog (AKT) expression. (A) The level of hTERT was examined by an enzyme-linked immunosorbant assay. (B) The relationship between hTERT and phosphorylated AKT (pAKT) was compared using a Spearman's correlation analysis. Error bar, SE. CG, chronic gastric mucosa without Helicobacter pylori (H. pylori); CAG, chronic active gastritis with H. pylori; CMG: chronic metaplastic gastritis without $H$. pylori; CGA, chronic gastritis with atypia without $H$.pylori. (A) Among the four mucosal categories, only CGA samples showed hTERT expression. (B) The levels of hTERT were correlated with the levels of pAKT.
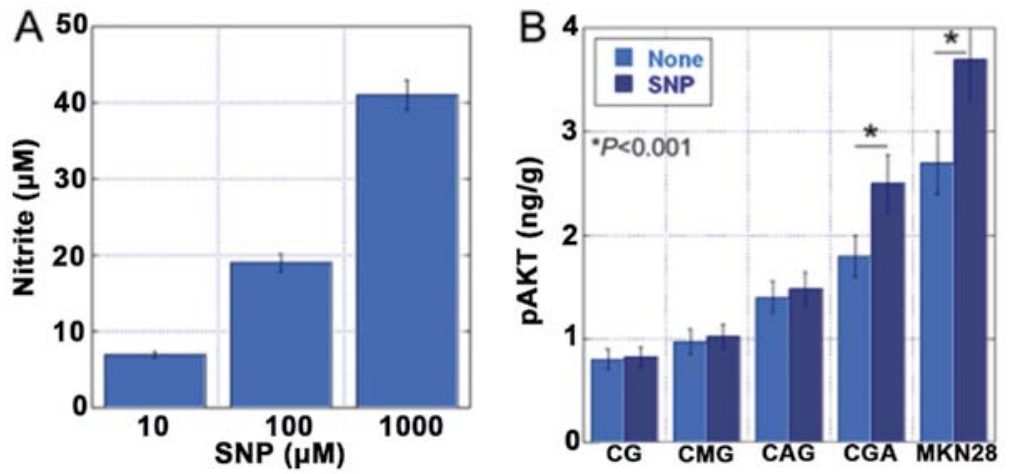

Figure 7. The phosphorylation of the v-akt murine thymoma viral oncogene homolog (AKT) in response to the NO level in the gastric mucosa. (A) The levels of nitrite generated by the treatment of cells with a NO donor, sodium nitroprusside (SNP). (B) Fresh tissues biopsy samples (n=3 in each category) were incubated with SNP $(100 \mu \mathrm{M})$, and the level of phosphorylated AKT (pAKT) was determined by an enzyme-linked immunosorbant assay. MKN28 cells are transformed gastric cells. Error bar, SE. CG, chronic gastric mucosa without Helicobacter pylori (H. pylori); CAG, chronic active gastritis with $H$. pylori; CMG, chronic metaplastic gastritis without $H$. pylori; CGA, chronic gastritis with atypia without $H$. pylori. The levels of pAKT were increased by SNP treatment in CGA samples and in MKN28 cancer cells, but not in the CG, CAG or CMG samples.

The gastric mucosa was classified into four categories: chronic gastric mucosa without Helicobacter pylori (H. pylori) (CG), chronic active gastritis with $H$. pylori (CAG), chronic metaplastic gastritis without $H$. pylori (CMG) and chronic gastritis with atypia without $H$. pylori (CGA) (Fig. 3). The levels of pAKT were associated with the levels of iNOS, NT and hTERT. In addition, the levels of pAKT, iNOS and NT increased in the order CG, CAG, CMG and CGA (Figs. 4-7). 


\section{Conclusions}

The role of AKT signal transduction in gastric cancer was discussed. It is important to analyze the role of AKT while taking into consideration the microenvironment and individual variations. The AKT signal transduction in gastric cancer may represent a biomarker for the diagnosis of gastric cancer and a target for treatment in the future.

\section{References}

1. Tokunaga E, Oki E, Egashira A, Sadanaga N, et al: Deregulation of the Akt pathway in human cancer. Curr Cancer Drug Targets 8: 27-36, 2008.

2. Stiles B, Gilman V, Khanzenzon N, et al: Essential role of AKT-1/protein kinase B alpha in PTEN-controlled tumorigenesis. Mol Cell Biol 22: 3842-3851, 2002.

3. Bellacosa A, Testa JR, Moore R and Larue L: A portrait of AKT kinases: human cancer and animal models depict a family with strong individualities. Cancer Biol Ther 3: 268-275, 2004

4. Manning BD and Cantley LC: AKT/PKB signaling: navigating downstream. Cell 129: 1261-1274, 2007.

5. Engelman JA, Luo J and Cantley LC: The evolution of phosphatidylinositol 3-kinases as regulators of growth and metabolism. Nat Rev Genet 7: 606-619, 2006.

6. Vivanco I and Sawyers CL: The phosphatidylinositol 3-Kinase AKT pathway in human cancer. Nat Rev Cancer 2 . 489-501, 2002.

7. Sukawa Y, Yamamoto H, Nosho K, et al: Alterations in the human epidermal growth factor receptor 2-phosphatidylinositol 3-kinase-v-Akt pathway in gastric cancer. World $\mathbf{J}$ Gastroenterol 18: 6577-6586, 2012.

8. Berg M and Soreide K: EGFR and downstream genetic alterations in KRAS/BRAF and PI3K/AKT pathways in colorectal cancer: implications for targeted therapy. Discov Med 14: 207-214, 2012.

9. Ng L, Poon RT and Pang R: Biomarkers for predicting future metastasis of human gastrointestinal tumors. Cell Mol Life Sci 70: 3631-3656, 2013.

10. Cheung M and Testa JR: Diverse mechanisms of AKT pathway activation in human malignancy. Curr Cancer Drug Targets 13: 234-244, 2013

11. Downward J: PI 3-kinase, Akt and cell survival. Semin Cell Dev Biol 15: 177-182, 2004.

12. Radisavljevic Z: AKT as locus of cancer angiogenic robustness and fragility. J Cell Physiol 228: 21-24, 2013.

13. Xu M and Mo YY: The Akt-associated microRNAs. Cell Mol Life Sci 69: 3601-3612, 2012.

14. Park BK, Zeng X and Glazer RI: Akt1 induces extracellular matrix invasion and matrix metalloproteinase- 2 activity in mouse mammary epithelial cells. Cancer Res 61: 7647-7653, 2001.
15. Kim D, Kim S, Koh H, et al: Akt/PKB promotes cancer cell invasion via increased motility and metalloproteinase production. FASEB J 15: 1953-1962, 2001.

16. Tanno S, Tanno S, Mitsuuchi Y, Altomare DA, Xiao GH and Testa JR: AKT activation up-regulates insulin-like growth factor I receptor expression and promotes invasiveness of human pancreatic cancer cells. Cancer Res 61: 589-593, 2001.

17. Grille SJ, Bellacosa A, Upson J, et al: The protein kinase Akt induces epithelial mesenchymal transition and promotes enhanced motility and invasiveness of squamous cell carcinoma lines. Cancer Res 63: 2172-2178, 2003.

18. Liu P, Cheng H, Roberts TM and Zhao JJ: Targeting the phosphoinositide 3-kinase pathway in cancer. Nat Rev Drug Discov 8: 627-644, 2009

19. Granville CA, Memmott RM, Gills JJ and Dennis PA: Handicapping the race to develop inhibitors of the phosphoinositide 3-kinase/Akt/mammalian target of rapamycin pathway. Clin Cancer Res 12: 679-689, 2006

20. Cully M, You H, Levine AJ and Mak TW: Beyond PTEN mutations: the PI3K pathway as an integrator of multiple inputs during tumorigenesis. Nat Rev Cancer 6: 184-192, 2006.

21. Markman B, Atzori F, Pérez-García J, Tabernero J and Baselga J: Status of PI3K inhibition and biomarker development in cancer therapeutics. Ann Oncol 21: 683-691, 2010.

22. Yuan R, Kay A, Berg WJ and Lebwohl D: Targeting tumorigenesis: development and use of mTOR inhibitors in cancer therapy. J Hematol Oncol 2: 45, 2009.

23. Li H, Zhao L, Yang Z, Funder JW and Liu JP: Telomerase is controlled by protein kinase Calpha in human breast cancer cells. J Biol Chem 273: 33436-33442, 1998.

24. Kang SS, Kwon T, Kwon DY and Do SI: Akt protein kinase enhances human telomerase activity through phosphorylation of telomerase reverse transcriptase subunit. J Biol Chem 274: 13085-13090, 1999.

25. Kim JH, Park SM, Kang MR, et al: Ubiquitin ligase MKRN1 modulates telomere length homeostasis through a proteolysis of hTERT. Genes Dev 19: 776-781, 2005.

26. Karamitopoulou E: Tumor budding cells, cancer stem cells and epithelial-mesenchymal transition-type cells in pancreatic cancer. Front Oncol 2: 209, 2013.

27. Liu Z, Li Q, Li K, et al: Telomerase reverse transcriptase promotes epithelial-mesenchymal transition and stem cell-like traits in cancer cells. Oncogene 32: 4203-4213, 2013.

28. Fidler IJ: The pathogenesis of cancer metastasis: the 'seed and soil' hypothesis revisited. Nat Rev Cancer 3: 453-458, 2003.

29. Claesson-Welsh L and Welsh M: VEGFA and tumour angiogenesis. J Intern Med 273: 114-127, 2013.

30. Karar J and Maity A: PI3K/AKT/mTOR pathway in angiogenesis. Front Mol Neurosci 4: 51, 2011

31. Sasaki T, Kuniyasu H,Luo Y, et al: AKT activation and telomerase reverse transcriptase expression are concurrently associated with prognosis of gastric cancer. Pathobiology 81: 36-41, 2014.

32. Sasaki T, Kuniyasu H, Luo Y, et al: Increased phosphorylation of AKT in high-risk gastric mucosa. Anticancer Res 33: 3295-3300, 2013 . 